\title{
Identification of chemoradiation-resistant osteosarcoma stem cells using an imaging system for proteasome activity
}

\author{
KEISUKE TAMARI $^{1,2^{*}}$, KAZUHIKO HAYASHI $^{1,2^{*}}$, HIDESHI ISHII ${ }^{2,3}$, YOSHIHIRO KANO ${ }^{2,4}$, \\ MASAMITSU KONNO ${ }^{2}$, KOICHI KAWAMOTO ${ }^{2,4}$, NAOHIRO NISHIDA ${ }^{2}$, JUN KOSEKI ${ }^{3}$, \\ TAKAHITO FUKUSUMI ${ }^{2,5}$, SHINICHIRO HASEGAWA ${ }^{2,4}$, HISATAKA OGAWA ${ }^{2,4}$, \\ ATSUSHI HAMABE ${ }^{2,4}$, MASAAKI MIYO ${ }^{2,4}$, KOZO NOGUCHI ${ }^{2,4}$, YUJI SEO ${ }^{1}$, \\ YUICHIRO DOKI $^{4}$, MASAKI MORI ${ }^{4}$ and KAZUHIKO OGAWA ${ }^{1}$ \\ Departments of ${ }^{1}$ Radiation Oncology, ${ }^{2}$ Frontier Science for Cancer and Chemotherapy, \\ ${ }^{3}$ Cancer Profiling Discovery, ${ }^{4}$ Gastroenterological Surgery, and ${ }^{5}$ Otorhinolaryngology-Head and \\ Neck Surgery, Osaka University, Graduate School of Medicine, Suita, Osaka 565-0871, Japan
}

Received June 28, 2014; Accepted August 12, 2014

DOI: 10.3892/ijo.2014.2671

\begin{abstract}
Osteosarcoma is the most common primary bone malignancy in pediatric and adolescent populations. Recurrence and metastatic potential can be due to a subpopulation of cells with stem cell-like characteristics, such as tumor-initiating cells (TICs), which maintain the capacity to regenerate entire tumors. Targeting the TICs in osteosarcoma is a promising avenue for the development of new therapies for this devastating disease. TICs are usually quiescent with a low protein turnover, decreased metabolism, and downregulation of proteasome activity. Recently, cancer cells with low proteasome activity have been identified as TICs in several types of cancer. We stably infected two osteosarcoma cell lines, MG-63 and $\mathrm{U} 2-\mathrm{OS}$, with an expression vector for a fusion protein between the green fluorescent protein, ZsGreen, and the C-terminal degron of the murine ornithine decarboxylase to monitor the $26 \mathrm{~S}$ proteasome activity in living cells. We separated the osteosarcoma cells with low proteasome activity using fluorescence-activated cell sorting (FACS) and verified whether these $\mathrm{ZsGreen}^{+}$cells had TIC-like properties. The $\mathrm{ZsGreen}^{+}$
\end{abstract}

Correspondence to: Dr K. Ogawa, Department of Radiation Oncology, Osaka University, Graduate School of Medicine, 2-2 (D10) Yamadaoka, Suita, Osaka 565-0871, Japan

E-mail: kogawa@radonc.med.osaka-u.ac.jp

Dr H. Ishii, Department of Cancer Profiling Discovery, Osaka University, Graduate School of Medicine, Center of Medical Innovation and Translational Research (0814B), Yamadaoka 2-2, Suita, Osaka 565-0871, Japan

E-mail: hishii@gesurg.med.osaka-u.ac.jp

*Contributed equally

Key words: osteosarcoma, cancer stem cells, proteasome activity cells showed enhanced sphere formation capacity and underwent asymmetric divisions into $\mathrm{ZsGreen}^{+}$and $\mathrm{ZsGreen}^{-}$cells, whereas $\mathrm{ZsGreen}^{-}$cells underwent only symmetric divisions into ZsGreen ${ }^{-}$cells. Moreover, the ZsGreen ${ }^{+}$cells were more chemo- and radioresistant. Thus, the present study demonstrated that chemoradiation-resistant TICs can be visualized by this system and suggested the rationale for further study of osteosarcoma stem cells.

\section{Introduction}

Osteosarcoma is the most common primary bone malignancy in children and young adults. Osteosarcoma occurs in the long bones of the limbs, particularly in the distal femur and proximal tibia. Osteosarcoma is a locally aggressive tumor and tends to produce early distant metastases, particularly to the lung. Before 1970, amputation was the only treatment for osteosarcoma patients and $80 \%$ patients died of metastatic disease (1). Since the 1970s, the combination of limb-sparing surgery and conventional chemotherapy agents, including methotrexate (MTX), cisplatin (CDDP), and doxorubicin, has been used to treat osteosarcoma. However, the 5-year patient survival has plateaued at $\sim 60-70 \%$ (2).

Tumors are organized into a hierarchy of heterogeneous cell populations. Recurrence and metastatic potential may be due to a subpopulation of cells with stem cell-like characteristics, such as cancer stem cells (CSCs) or tumor-initiating cells (TICs), which maintain the capacity to regenerate entire tumors (3). Targeting the TICs in osteosarcoma may be a promising avenue to explore for the development of new therapies for this devastating disease.

Increasing evidence of the existence of TICs in patients with osteosarcoma has been reported. Identification of osteosarcoma TICs has been performed using CD133 $(4,5)$, side populations $(6,7)$, PKH26 (8), ALDH1 $(9,10)$, and the promoter reporter assays of hTERT (11) and Oct3/4 (12). TIC-enriched osteosarcoma cell populations exhibit capacity for self-renewal, multilineage differentiation, tumorigenicity, 
and chemo- and radioresistance. Furthermore, TICs are usually quiescent, with a low protein turnover, decreased metabolism, and downregulation of proteasome activity. The ubiquitinproteasome system is the major non-lysosomal system for the degradation of intracellular proteins. Recently, cancer cells with low proteasome activity have been identified as TICs in patients with breast cancer, glioma (13), pancreatic cancer (14), and esophageal cancer (15) using a fluorescence marker system for the level of proteasome activity. However, no study has reported the identification of TICs in human osteosarcoma cell lines on the basis of low proteasome activity.

Here we showed that a small subpopulation of osteosarcoma cells with low proteasome activity had TIC-like properties. Human osteosarcoma cell lines were transfected with a retroviral vector that monitored proteasome activity using a fluorescent protein (ZsGreen). We isolated a fraction of cells with low proteasome activity from these human osteosarcoma cell lines and identified these cells to have tumor-initiating capacity.

\section{Materials and methods}

Cell culture. Human osteosarcoma cell lines U2-OS and MG-63 were purchased from the American Type Culture Collection (Manassas, VA, USA) and cultured in Dulbecco's modified Eagle's medium (Invitrogen) supplemented with $10 \%$ fetal bovine serum (Gibco), penicillin, and streptomycin (Sigma). All cells were grown in a humidified incubator at $37^{\circ} \mathrm{C}$ with $5 \% \mathrm{CO}_{2}$.

Generation of stable cell lines expressing ZsGreen-cODC fusion proteins using retroviral transduction. The retroviral expression vector pQCXIN-ZsGreen-cODC, containing green fluorescence ZsGreen-labeled degron ODC, was kindly provided by Dr Shinji Tanaka. The retroviral vector was transfected into platinum A (Plat-A) to generate a retrovirus. The vector was transfected into Plat-A retroviral packaging cells using FuGENE 6 (Promega); the virus collected from the supernatant of Plat-A was used to infect osteosarcoma cells. The stable transfectants were selected with Geneticin (Invitrogen) and the accumulation of ZsGreen-labeled degron ODC protein was monitored by fluorescence microscopy and flow cytometry. To determine if this was a stable transfection, the cells were exposed to proteasome inhibitor MG-132 (Wako, Japan) for $12 \mathrm{~h}$.

Time-lapse analysis. After FACS, $\mathrm{ZsGreen}^{+}$cells were separately plated at a density of $10^{4}$ cells in $35-\mathrm{mm}$ dishes in Dulbecco's modified Eagle's medium (Invitrogen) supplemented with $10 \%$ fetal bovine serum (Gibco) and Pen/Strep (Sigma). After incubation in $5 \% \mathrm{CO}_{2}$ at $37^{\circ} \mathrm{C}$ overnight, cell attachment was confirmed. Image analysis was performed using a FV1200 confocal microscope (Olympus).

Flow cytometry. We used a FACS Aria II (BD Biosciences, San Jose, CA, USA) for cell sorting. Osteosarcoma cells were washed with phosphate-buffered saline (PBS) and enzymatically dissociated with $0.05 \%$ trypsin-EDTA (Invitrogen). Cells were gently triturated and filtered through cell strainer caps (35- $\mu \mathrm{m}$ mesh) to obtain a single cell suspension $\left(\sim 1 \times 10^{7}\right.$ cells $/ \mathrm{ml})$. The presence of ZsGreen allows the selection of cells with low proteasome activity using flow cytometry with standard fluorescein isothiocyanate (FITC) for cell sorting.

Measurement of proteasome activity. Using Proteasome-Glo ${ }^{\mathrm{TM}}$ cell-based assays (Promega), the chymotrypsin-like, trypsinlike, and caspase-like activities were measured according to the manufacturer's protocol. Proteasome-Glo ${ }^{\mathrm{TM}}$ buffer was mixed with luciferin detection reagent, and the substrate was added to the mixture and incubated at room temperature for $1 \mathrm{~h}$. An equal volume of proteasome-Glo ${ }^{\mathrm{TM}}$ reagent was added to the samples and further incubated for $15 \mathrm{~min}$. The luminogenic substrate containing the Suc-LLVY sequence was recognized by the proteasome. Following the proteasome cleavage, the substrate for luciferase (aminoluciferin) was released, allowing the luciferase reaction to proceed and produce light. The luminescence was measured using a luminometer (Thermo Fisher Scientific, Waltham, MA, USA).

Sphere formation assay. ZsGreen ${ }^{+}$and $\mathrm{ZsGreen}^{-}$cells were sorted by FACS, plated separately at a density of 10,000 cells on low-attachment 6-well plates (Costar, Corning, NY, USA), and incubated in serum-free Dulbecco's modified Eagle's medium/F12 medium (Invitrogen) supplemented with b-FGF, EGF (Sigma), and N2 (Wako). After 14 days, spheres with diameters $>100 \mu \mathrm{m}$ were counted.

Tumorigenicity assay. Our animal studies were approved by the Animal Experiments Committee of Osaka University (Suita, Japan). Following FACS, the portions containing $1 \times 10^{3}, 1 \times 10^{4}$ and $1 \times 10^{5}$ cells were mixed with BD Matrigel (Becton-Dickinson, Franklin Lakes, NJ, USA) at a 1:1 ratio and were subcutaneously injected into 4- to 5-week-old female NOD/SCID mice (Charles River, Japan). The animals were anesthetized with isofluorane and maintained under sterile airflow conditions during the experiments.

Cell proliferation assay. Proliferation assays for the U2-OS and MG-63 cells treated with MTX and CDDP (both purchased from Wako, Japan) were performed to test chemosensitivity. Briefly, a total of $2 \times 10^{3}$ of both ZsGreen ${ }^{+}$and ZsGreen cells were sorted, individually seeded into 96-well plates (Corning), and treated with varying concentrations of the drugs for $72 \mathrm{~h}$. The assay was performed using a commercially available cell counting kit-8 (Dojindo, Japan). The remaining viable cell count was determined by measuring the absorbance at $450 \mathrm{~nm}$ using an Enspire plate reader (Perkin-Elmer).

Clonogenic survival assay. The appropriate number of $\mathrm{ZsGreen}^{+}$and ZsGreen ${ }^{-}$cells were sorted, individually seeded in 6-cm dishes, and exposed to radiation at $0,2,4,6$, and 8 Gy by Cs-137 gamma irradiation generated using a Gammacell 40 Exactor (MDS Nordion, Ottawa, Canada). After incubation for 14 days, the colonies were fixed and stained with crystal violet. Colonies containing $>50$ cells were counted as survivors. At least three parallel samples were scored in three to five repetitions conducted for each irradiation condition.

Statistical analysis. Data are expressed as means \pm SDs. Statistically significant differences were determined by 

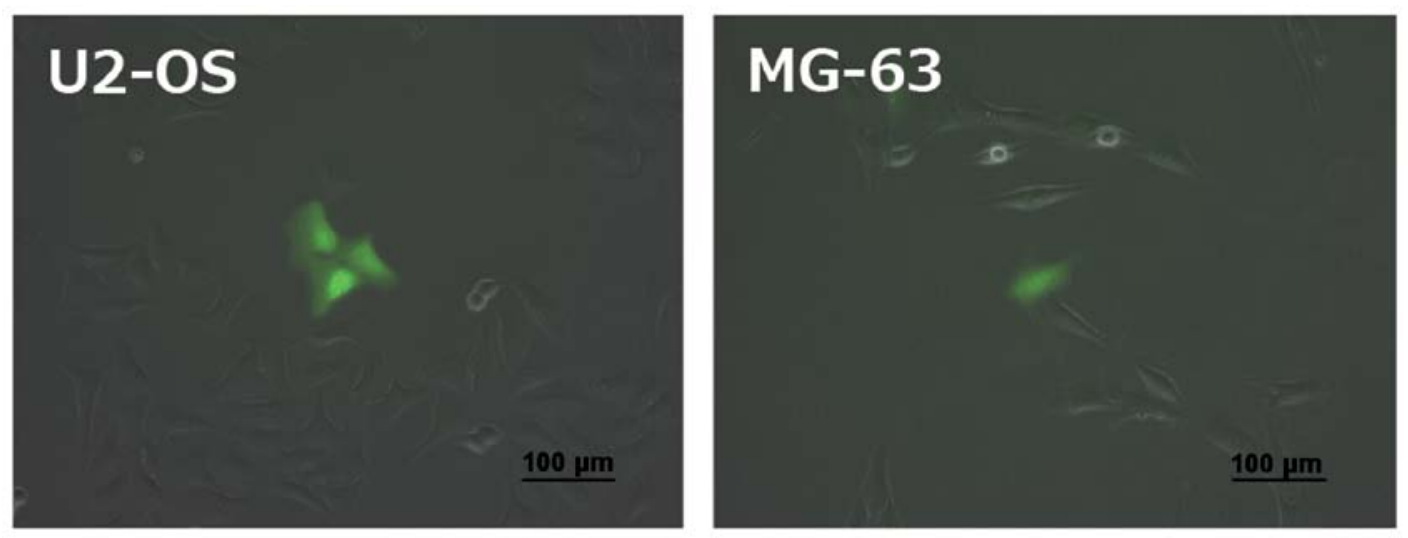

Figure 1. Establishment of osteosarcoma cell lines transfected with a proteasome sensor vector. The frequency of cells with accumulation of ZsGreen-ODC protein in human osteosarcoma cultures (bar, $100 \mu \mathrm{m}$ ).

A
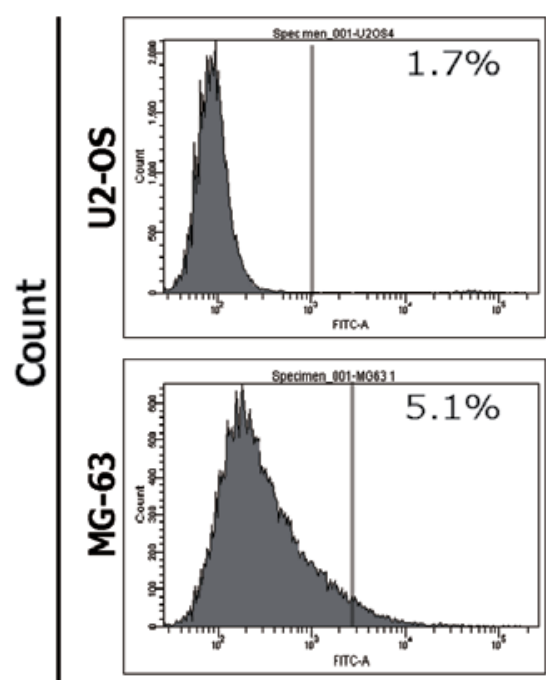

MG-132
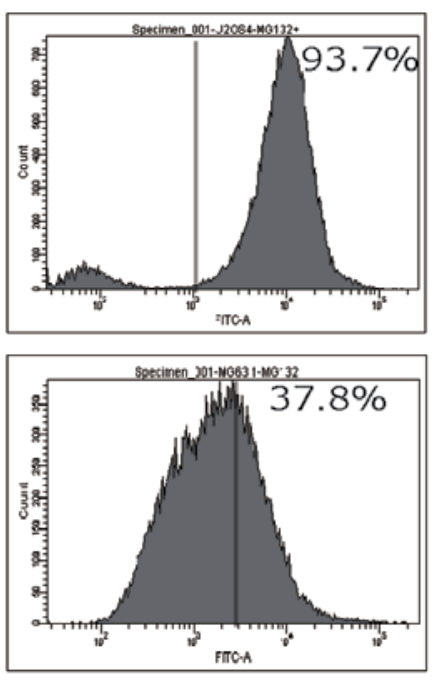

B

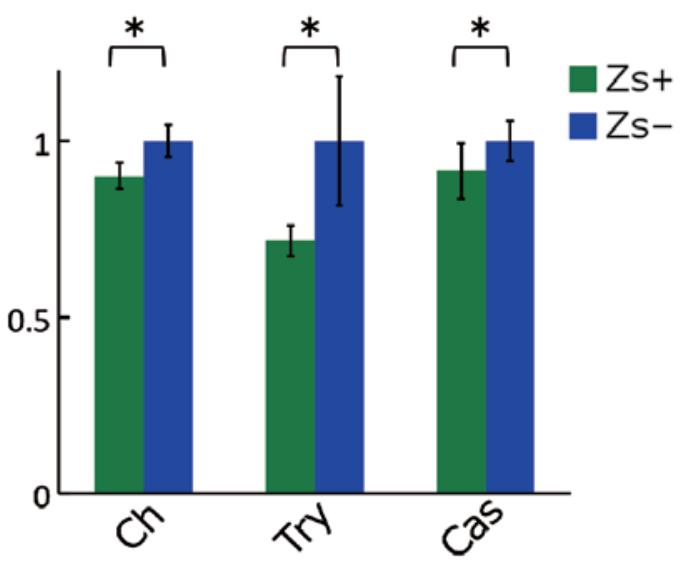

\section{ZsGreen}

Figure 2. ZsGreen ${ }^{+}$cells showing low proteasome activity. (A) Fractions of ZsGreen ${ }^{+}$cells were measured by fluorescence-activated cell sorting (FACS) (left). When two cell lines are incubated with the proteasome inhibitor MG-132 overnight all cells accumulate the fusion protein. (B) ZsGreen ${ }^{+}$cells of MG-63 showing low proteasome activity. The value is standardized with ZsGreen cells. Ch, chymotrypsin-like activity. Try, trypsin-like activity. Cas, caspase-like activity. ${ }^{*} \mathrm{p}<0.05$. Error bars indicate means \pm SD.

two-way ANOVA analysis and Student's t-test, where appropriate, and were defined as $\mathrm{p}<0.05$.

\section{Results}

Establishment of osteosarcoma cell lines transfected with proteasome sensor vector. To monitor proteasome activity, two osteosarcoma cell lines, U2-OS and MG-63, were stably transfected with retroviral vector pQCXIN-ZsGreen-cODC. After geneticin selection and single cell cloning, we established two osteosarcoma cell lines transfected with a proteasome sensor vector. We divided these cells into $\mathrm{ZsGreen}^{+}$and $\mathrm{ZsGreen}^{-}$groups according to their fluorescence intensity. We could easily identify ZsGreen ${ }^{+}$cells (Fig. 1). The FACS analysis showed that the fractions of $\mathrm{ZsGreen}^{+}$cells were $1.7 \%$ in U2-OS and 5.1\% in MG-63 (Fig. 2A). To validate the protea- some activity monitoring, we performed proteasome inhibition of these established cell lines with MG-132. Fig. 2A shows that proteasome inhibition dramatically increased the fraction of $\mathrm{ZsGreen}^{+}$cells from 1.7 to $93.7 \%$ in U2-OS and from 5.1 to $37.8 \%$ in MG-63. Furthermore, we directly measured proteasome activity, such as trypsin-like, chymotrypsin-like, and caspase-like activities, using Proteasome Glo; this showed that $\mathrm{ZsGreen}^{+}$cells had lower proteasome activity compared with ZsGreen ${ }^{-}$cells in MG-63 (Fig. 2B), but not in U2-OS (data not shown).

$\mathrm{ZsGreen}^{+}$cells regenerate $\mathrm{ZsGreen}^{+}$and ZsGreen cells. TICs have self-renewal and multilineage differentiation capacity. To test this, we separately cultured $\mathrm{ZsGreen}^{+}$and $\mathrm{ZsGreen}^{-}$cells after cell sorting for U2-OS. Time-lapse imaging showed asymmetric divisions of the $\mathrm{ZsGreen}^{+}$cells into $\mathrm{ZsGreen}^{+}$ 
A


\section{ZsGreen}

Figure 3. ZsGreen ${ }^{+}$cells divided into ZsGreen ${ }^{+}$and ZsGreen ${ }^{-}$cells. (A) Time-lapse imaging shows the asymmetric division of ZsGreen ${ }^{+}$cells from U2-OS (B) Immediately after sorting, ZsGreen ${ }^{+}$and ZsGreen ${ }^{-}$cells of U2-OS were separately cultured. After 7 days, fluorescence-activated cell sorting (FACS) analysis revealed that ZsGreen ${ }^{+}$cells divide into $\mathrm{ZsGreen}^{+}$and $\mathrm{ZsGreen}^{-}$cells, whereas ZsGreen` cells divide into only ZsGreen cells.

and ZsGreen ${ }^{-}$cells (Fig. 3A), whereas the ZsGreen cells did not divide into ZsGreen ${ }^{+}$cells. Similarly, the FACS analysis showed that the ZsGreen ${ }^{+}$cells of U2-OS regenerated into $\mathrm{ZsGreen}^{+}$and $\mathrm{ZsGreen}^{-}$cells, whereas the $\mathrm{ZsGreen}^{-}$cells divided into only ZsGreen cells (Fig. 3B). These findings indicate that the $\mathrm{ZsGreen}^{+}$population had the capacity for self-renewal and multilineage differentiation. However, the ZsGreen $^{+}$and ZsGreen ${ }^{-}$cells of MG-63 divided into both $\mathrm{ZsGreen}^{+}$and $\mathrm{ZsGreen}^{-}$cells, which indicated that both the populations had the capacity for differentiation.

Sphere-forming capacity. To evaluate self-renewal, we evaluated the sphere-forming capacity of $\mathrm{ZsGreen}^{+}$and $\mathrm{ZsGreen}^{-}$cells. A total of $10,000 \mathrm{ZsGreen}^{+}$and $\mathrm{ZsGreen}^{-}$ cells were sorted and cultured in serum-free conditions. The frequencies of sphere formation for $\mathrm{ZsGreen}^{+}$and $\mathrm{ZsGreen}^{-}$ were 0.16 and $0.013 \%(\mathrm{p}<0.001)$ in $\mathrm{U} 2-\mathrm{OS}$ and 0.23 and $0.097 \%$ $(\mathrm{p}=0.027)$ in MG-63 cells (Fig. 4A and B), respectively. The $\mathrm{ZsGreen}^{+}$cells showed a high frequency of sphere formation compared with the ZsGreen ${ }^{-}$cells.

Tumorigenicity assay. TICs are primarily characterized by the ability to form tumors in NOD/SCID mice. To determine whether ZsGreen ${ }^{+}$cells were more tumorigenic than $\mathrm{ZsGreen}^{-}$ cells in vivo, we injected both the populations separately into NOD/SCID mice. After 3 months, no tumors were observed (Table I).

Treatment resistance. To test chemoresistance, $\mathrm{ZsGreen}^{+}$and ZsGreen ${ }^{-}$cells from two osteosarcoma cell lines were exposed to MTX and CDDP. The difference in chemoresistance between the $\mathrm{ZsGreen}^{+}$and $\mathrm{ZsGreen}^{-}$cells was assessed using 
A
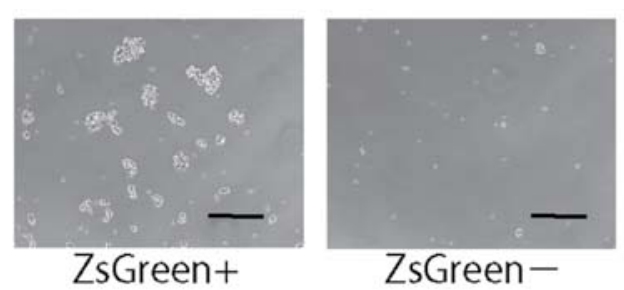

B

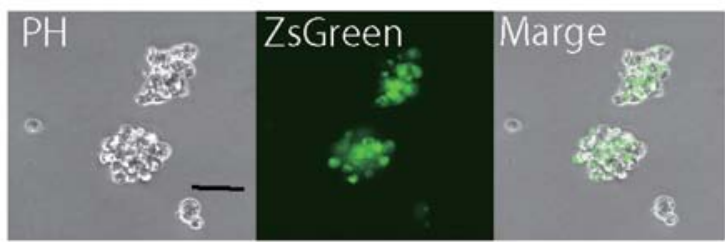

C
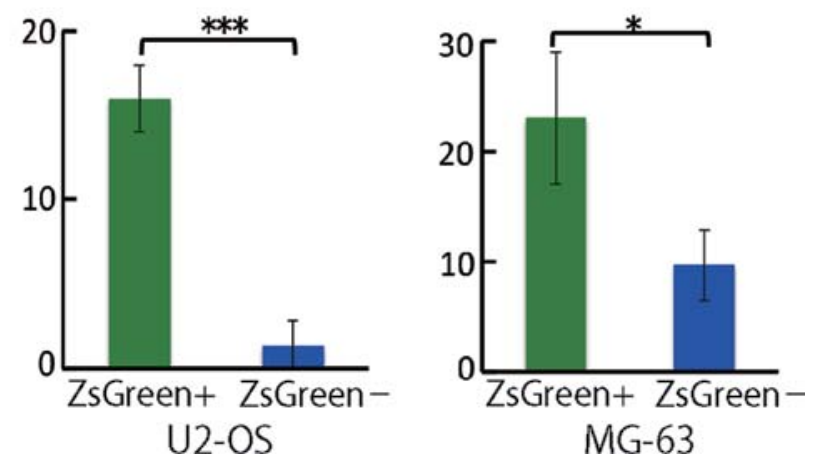

Figure 4. Sphere-forming assay. Immediately after sorting, a total of 10,000 $\mathrm{ZsGreen}^{+}$and ZsGreen ${ }^{-}$cells are separately cultured in serum-free medium supplemented with b-FGF, b-EGF, and N2. After 14 days, a strong sphereforming capacity is observed in $\mathrm{ZsGreen}^{+}$cells. (A) Microscopic images in the low-power field (bar, $300 \mu \mathrm{m}$ ). (B) Microscopic images in the high-power field show that ZsGreen ${ }^{+}$cells primarily comprise spheres (bar, $100 \mu \mathrm{m}$ ). (C) Quantitative analysis of the number of spheres. Data are presented as means $\pm \mathrm{SD}\left(\mathrm{t}\right.$-test: $\left.{ }^{*} \mathrm{p}<0.05,{ }^{* * *} \mathrm{p}<0.001\right)$.
Table I. Tumorigenesis of $\mathrm{ZsGreen}^{+}$and $\mathrm{ZsGreen}^{-}$cells of U2-OS in NOD/SCID mice.

\begin{tabular}{lccc}
\hline & \multicolumn{3}{c}{ No. of cells per injection } \\
\cline { 2 - 4 } & $1 \times 10^{3}$ & $1 \times 10^{4}$ & $1 \times 10^{5}$ \\
\hline ZsGreen $^{+}$cells & $0 / 6$ & $0 / 6$ & $0 / 6$ \\
ZsGreen $^{-}$cells & $0 / 6$ & $0 / 6$ & $0 / 6$
\end{tabular}

$\mathrm{ZsGreen}^{+}$and $\mathrm{ZsGreen}^{-}$cells were isolated separately and injected into the subcutaneous space of NOD/SCID mice. Tumor formation was observed for 12 weeks after injection.

the cell proliferation assay. Of the two cell lines, the ZsGreen ${ }^{+}$ cells exhibited significant resistance to MTX, but not to CDDP (Fig. 5A).

Next, to evaluate radioresistance, we performed clonogenic survival assays for ZsGreen ${ }^{+}$and $\mathrm{ZsGreen}^{-}$cells of the two cell lines. As shown in Fig. 5B, ZsGreen ${ }^{+}$cells were more radioresistant than ZsGreen ${ }^{-}$cells in both cell lines. These results indicated that the $\mathrm{ZsGreen}^{+}$cells were resistant to chemotherapy and radiotherapy.

\section{Discussion}

Our findings indicate that low proteasome activity can serve as a marker to identify a subpopulation of TIC cells in osteosarcoma. The ZsGreen ${ }^{+}$cells displayed several features that are typically observed in TICs, including self-renewal, generation of differentiated progeny, and treatment resistance. We observed that the fractions of $\mathrm{ZsGreen}^{+}$cells were 1.7 and
A

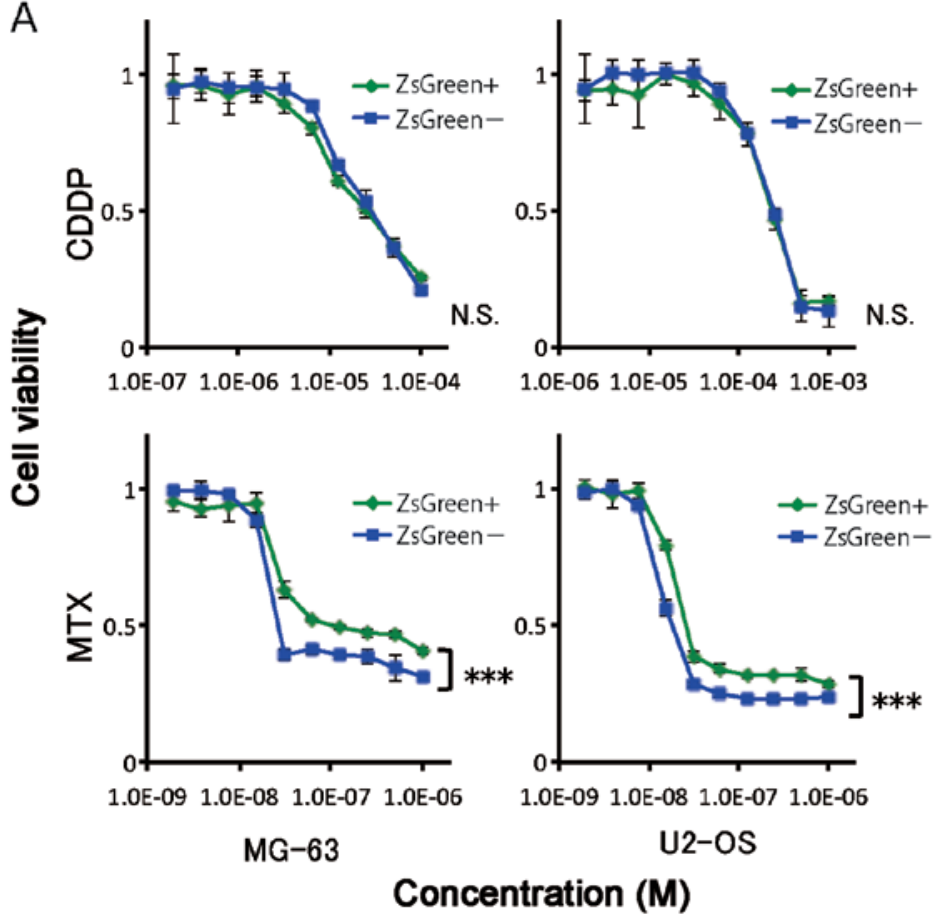

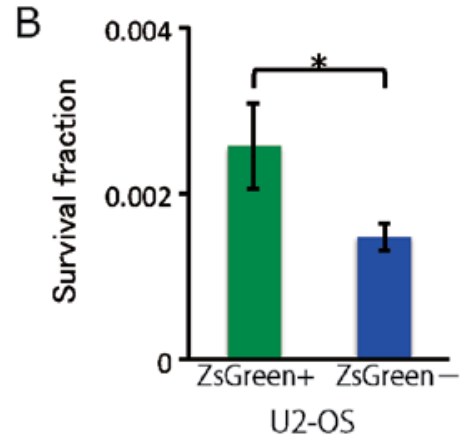

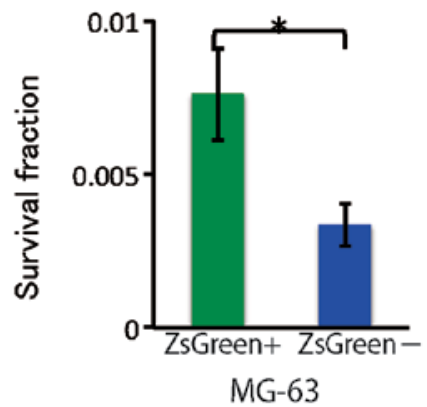

Figure 5. Treatment resistance. (A) A cell proliferation assay reveals that compared with ZsGreen ${ }^{-}$cells, ZsGreen $^{+}$cells are more chemoresistant to MTX, but not to CDDP. Data are presented as means \pm SDs (two-way ANOVA, ${ }^{* * *}$ p $<0.001$ ). (B) A clonogenic survival assay reveals that ZsGreen ${ }^{+}$cells are more radioresistant than ZsGreen ${ }^{-}$cells after $8 \mathrm{~Gy}$ of irradiation. Data are presented as means $\pm \mathrm{SD}\left(\mathrm{t}\right.$-test: $\left.{ }^{* *} \mathrm{p}<0.01\right)$. 
$5.1 \%$ in the U2-OS and MG-63 cells, respectively. Previous reports have shown that the fractions of $\mathrm{ZsGreen}^{+}$cells were $<4 \%$ in U87MG (13) and 0.5\% in Panc-1 (14). The proteasome activity of $\mathrm{ZsGreen}^{+}$cells are lower than $\mathrm{ZsGreen}^{-}$cells in MG-63, whereas no difference was found in U2-OS; this is likely because this proteasome activity assay can detect partial proteasome activity. The sphere-forming capacity in established osteosarcoma cell lines has been previously demonstrated $(4,8,9,11,12)$. Interestingly, Honoki et al showed that the frequency of sphere formation was $0.25 \%$ in ALDH1-positive MG-63 cells (9). Moreover, primary cultures of osteosarcoma tended to have enhanced sphere-forming capacities $(5,8,16)$.

Although sphere-forming capacity may be a good in vitro assay to study osteosarcoma TICs, the gold standard to identify TICs is the formation of tumors after limiting dilution transplantation in immunodeficient mice. There was no tumor formation when we performed limiting dilution transplantation in NOD/SCID mice. Wang et al also showed that the TIC-like populations (high ALDH activity) of the osteosarcoma cell lines did not form tumors, but they did show enhanced tumor formation in populations with high ALDH activity obtained from a xenograft (10). Similar to the observation in this study, the population with low proteasome activity in osteosarcoma cells in vivo may be more tumorigenic. We could not inoculate a larger number of cells, such as $10^{6}$ cells, because of the extensive long time required for the sorting process.

We studied treatment resistance of the $\mathrm{ZsGreen}^{+}$cells and found that they were chemo- and radioresistant. The mechanism by which treatment resistance occurs in TICs has not been elucidated. Di Fiore et al manufactured novel TIC-enriched cell lines such as 3AB-OS that were irreversibly selected from MG-63 cells after long exposure with 3-aminobenzamide (3AB) and expressed higher levels of the drug resistance marker ABCG2 (17). Furthermore, Honoki et al showed that the high ALDH1 population of MG-63 enhanced the resistance to CDDP and doxorubicin (9). Fujiawa et al recently found that miR-133a expression was correlated with chemo-resistance (18).

In conclusion, osteosarcoma cells with low proteasome activity had TIC properties, including sphere formation and chemo- and radioresistance. This result may lead to new approaches for the development of a more specific therapy and for an improvement in prognosis.

\section{Acknowledgements}

We thank Dr Shinji Tanaka at Department of Hepatobiliary and Pancreatic Surgery, Graduate School of Medicine, Tokyo Medical and Dental University for pQCXIN-ZsGreen-cODC plasmid. This study was supported in part by a Grant-in-Aid for Scientific Research and a grant from the Platform for Drug Discovery, Informatics, and Structural Life Science, from the Ministry of Education, Culture, Sports, Science and Technology; a Grant-in-Aid from the Third Comprehensive 10-Year Strategy for Cancer Control, Ministry of Health, Labour and Welfare, Japan. H.I., M.K., N.N., and J.K. received partial support from Chugai Co., Ltd., Yakult Honsha Co., Ltd., Merck Co., Ltd. and Taiho Therapeutic Co., Ltd., through institutional endowments.

\section{References}

1. Marcove RC, Miké V, Hajek J V, Levin AG and Hutter RV: Osteogenic sarcoma under the age of twenty-one. A review of one hundred and forty-five operative cases. J Bone Joint Surg Am 52: 411-423, 1970.

2. Gill J, Ahluwalia MK, Geller D and Gorlick R: New targets and approaches in osteosarcoma. Pharmacol Ther 137: 89-99, 2013.

3. Meacham CE and Morrison SJ: Tumour heterogeneity and cancer cell plasticity. Nature 501: 328-337, 2013.

4. Tirino V, Desiderio V, d'Aquino R, et al: Detection and characterization of CD133 ${ }^{+}$cancer stem cells in human solid tumors. PLoS One 3: e3469, 2008.

5. Tirino V, Desiderio V, Paino F, et al: Human primary bone sarcomas contain $\mathrm{CD} 133^{+}$cancer stem cells displaying high tumorigenicity in vivo. FASEB J 25: 2022-2030, 2011.

6. Murase M, Kano M, Tsukahara T, et al: Side population cells have the characteristics of cancer stem-like cells/cancer-initiating cells in bone sarcomas. Br J Cancer 101: 1425-1432, 2009.

7. Yang M, Yan M, Zhang R, Li J and Luo Z: Side population cells isolated from human osteosarcoma are enriched with tumorinitiating cells. Cancer Sci 102: 1774-1781, 2011.

8. Rainusso N, Man T-K, Lau CC, et al: Identification and gene expression profiling of tumor-initiating cells isolated from human osteosarcoma cell lines in an orthotopic mouse model. Cancer Biol Ther 12: 278-287, 2011.

9. Honoki K, Fujii H, Kubo A, Kido A, Mori T, Tanaka Y and Tsujiuchi T: Possible involvement of stem-like populations with elevated ALDH1 in sarcomas for chemotherapeutic drug resistance. Oncol Rep 24: 501-505, 2010.

10. Wang L, Park P, Zhang H, La Marca F and Lin C-Y: Prospective identification of tumorigenic osteosarcoma cancer stem cells in OS99-1 cells based on high aldehyde dehydrogenase activity. Int J Cancer 128: 294-303, 2011.

11. Yu L, Liu S, Zhang C, et al: Enrichment of human osteosarcoma stem cells based on hTERT transcriptional activity. Oncotarget 4: 2326-2338, 2013

12. Levings PP, McGarry S V, Currie TP, et al: Expression of an exogenous human Oct-4 promoter identifies tumor-initiating cells in osteosarcoma. Cancer Res 69: 5648-5655, 2009.

13. Vlashi E, Kim K, Lagadec C, et al: In vivo imaging, tracking, and targeting of cancer stem cells. J Natl Cancer Inst 101: 350-359, 2009.

14. Adikrisna R, Tanaka S, Muramatsu S, et al: Identification of pancreatic cancer stem cells and selective toxicity of chemotherapeutic agents. Gastroenterology 143: 234-245.e7, 2012.

15. Kano Y, Konno M, Kawamoto K, et al: Novel drug discovery system for cancer stem cells in human squamous cell carcinoma of the esophagus. Oncol Rep 31: 1133-1138, 2014.

16. Ying M, Liu G, Shimada H, et al: Human osteosarcoma CD49f(-)CD133(+) cells: impaired in osteogenic fate while gain of tumorigenicity. Oncogene 32: 4252-4263, 2013.

17. Di Fiore R, Santulli A, Ferrante RD, et al: Identification and expansion of human osteosarcoma-cancer-stem cells by long-term 3-aminobenzamide treatment. J Cell Physiol 219: 301-313, 2009.

18. Fujiwara T, Katsuda T, Hagiwara K, et al: Clinical relevance and therapeutic significance of microRNA-133a expression profiles and functions in malignant osteosarcoma-initiating cells. Stem Cells 32: 959-973, 2014. 